\title{
Analysing Wear in Carpets By Detecting Varying Local Binary Patterns
}

\author{
S. A. Orjuela ${ }^{a}$, E. Vansteenkiste ${ }^{a}$, F. Rooms ${ }^{a}$, S. De Meulemeester ${ }^{b}$, R. de $\operatorname{Keyser}^{c}$ and \\ W.Philips ${ }^{a}$ \\ ${ }^{a}$ Department of Telecommunications and Information Processing (TELIN), IPI, IBBT, Ghent \\ University, Sint-Pietersnieuwstraat 41, B-9000 Gent, Belgium; \\ ${ }^{b}$ Department of Textile, Ghent University, Belgium; \\ ${ }^{c}$ Department of Electrical energy, Systems and Automation, Ghent University, Belgium
}

\begin{abstract}
Currently, carpet companies assess the quality of their products based on their appearance retention capabilities. For this, carpet samples with different degrees of wear after a traffic exposure simulation process are rated with wear labels by human experts. Experts compare changes in appearance in the worn samples to samples with original appearance. This process is subjective and humans can make mistakes up to $10 \%$ in rating. In search of an objective assessment, research using texture analysis has been conducted to automate the process. Particularly, Local Binary Pattern (LBP) technique combined with a Symmetric adaptation of the KullbackLeibler divergence (SKL) are successful for extracting texture features related to the wear labels either from intensity and range images. We present in this paper a novel extension of the LBP techniques that improves the representation of the distinct wear labels. The technique consists in detecting those patters that monotonically change with the wear labels while grouping the others. Computing the SKL from these patters considerably increases the discrimination between the consecutive groups even for carpet types where other LBP variations fail. We present results for carpet types representing 72\% of the existing references for the EN1471:1996 European standard.
\end{abstract}

Keywords: LBP techniques, Image Analysis, Wear Analysis, Texture Analysis

\section{INTRODUCTION}

Nowadays, apart from other benefits, carpet customers are highly interested in fashioning indoor settings and carpet industries are motivated to certify the quality of their products based on aesthetics. A carpet in the market is labeled with a number, named 'wear label', which indicates an overall grade of the expected change in appearance after one year of installation. To do this, carpet samples of new products are first subjected to a mechanical simulation of traffic exposure. ${ }^{1}$ Afterwards, the new appearance is evaluated comparing changes in pattern,color, hairiness, thickness and structure with the appearance of an original sample, meaning a sample not exposed to any wear process. This comparison is currently performed by at least three human experts. In America, the comparison is performed by using photographs whereas physical samples previously labeled are used in Europe. ${ }^{2,3}$ Manufacturers however require a more objective assessment because the human assessment is often too subjective. Much effort has been devoted to automate this rating process, initially on techniques extracting texture features from digital images, ${ }^{4-12}$ and lately combining features from digital and range images, ${ }^{13-16}$ where a range image represents the depth information of the surface of a carpet. The range images are obtained with a 3D scanner designed specifically for scanning carpets. ${ }^{12}$

Recently, Local Binary Pattern (LBP) techniques have proved to be successful for extracting features representing the degree of wear in carpets as well as to perform better than other texture analysis techniques

Further author information: (Send correspondence to S. A. Orjuela)

S. A. Orjuela is supported by a grant number 20070207 of the 'LASPAU' Academic and Professional Programs for the Americas in agreement with COLCIENCIAS and Universidad Antonio Nariño, Colombia: E-mail: seraleov@telin.ugent.be, Telephone: +32 92643415 
like co-occurrence matrices and Laws energy measures. ${ }^{12,13,15}$ Particularly, using an extension of the known rotational Local Binary Pattern (LBPROT) ${ }^{17}$ technique named LBPRMC (from Local Binary Pattern Rotational, Mirrored and Complemented invariant) has shown that grouping patterns increases the linear rank of the features related to the degree of wear as well as the discrimination between consecutive degrees of wear. ${ }^{12}$

To further increase the discrimination between consecutive degrees of wear we present a new extension of the LBP technique based in detecting patters that change with the wear labels and grouping the others. Like in a previous approach, ${ }^{14}$ the technique has been tested in both intensity and range images from a database composed of samples from the EN1471:1996 standard. ${ }^{3}$ The results show that the discriminance between consecutive degrees of wear increases by reducing the noise given by the patters that do not change with the wear labels.

The paper is organized as follows. In Section 2, we present the set up for performing the experiment and the description of the database used. In Section 3 we describe the local binary pattern and its extensions. In Section 4, we report the results and in Section 5, findings are discussed and conclusions are drawn.

\section{MATERIALS}

The experiments were performed on eight types of reference sets provided from the EN1471:1996 standard, ${ }^{3}$ each containing a collection of samples of a particular type of carpet priorly subjected to different revolutions on the Vetterman test, a traffic simulator tester. ${ }^{1}$ Each reference includes eight labeled samples of transitional degrees of wear. These sets do not include wear label 5 and the wear labels vary from 4.5 to 1 , with steps of half point. Wear label 4.5 represents a minor change in appearance and wear label 1 a severe change. Currently, eleven types of carpets have been established for the EN1471:1996 European standard. This selection is based on a combination of characteristics in the carpet construction such as pile/surface fiber, type of manufacturer, secondary backing among others. Thus, based carpet construction this study cover $72 \%$ of the existing references for the EN1471:1996 European standard by using eight types from the eleven.

To compose the database of intensity images, the samples were vacuumed and five photographs were taken per sample, each one with a size of $720 \times 576$ pixels corresponding to $17.28 \mathrm{~cm} \times 13.82 \mathrm{~cm}$ of the surface. Thus, one pixel represents a surface with size of $0.24 \mathrm{~mm} \times 0.24 \mathrm{~mm}$ of a carpet sample. Gray images representing the intensity of the texture are used from these photographs. These photographs were converted from the RGB-model to the YUV-model, with values from 1 to 256 , to use an intensity component more shape representative. ${ }^{18}$

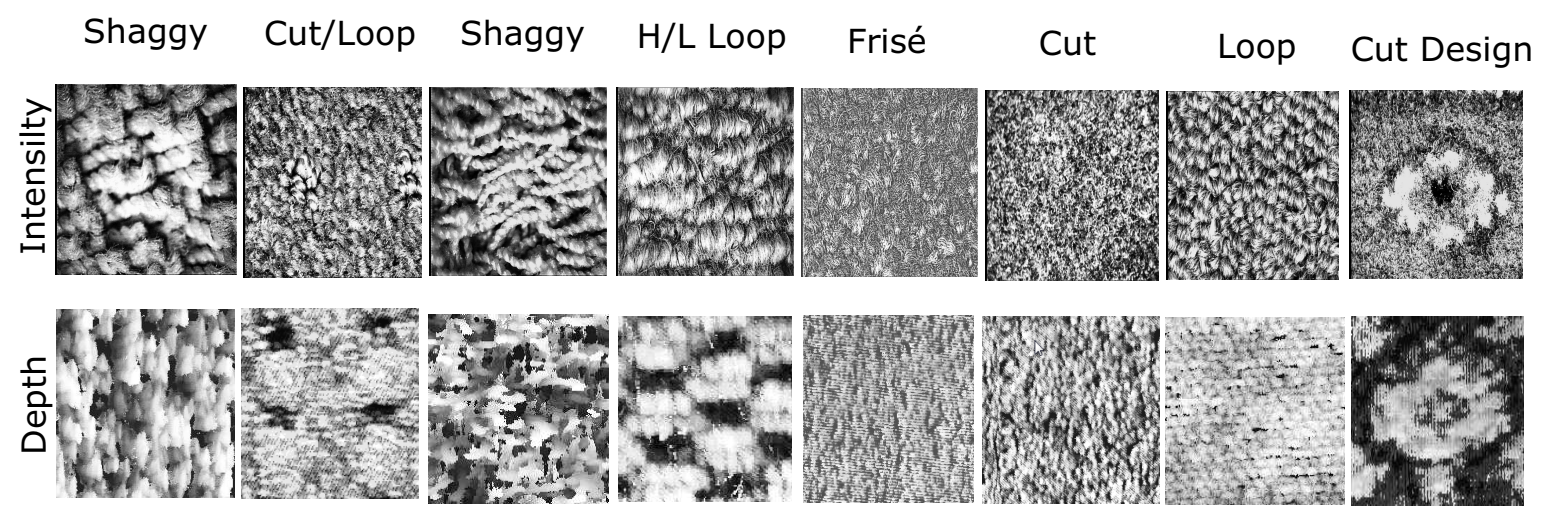

Figure 1: Samples of images from the database used in this approach.

To compose the database of range images, the samples were also scanned with the 3D scanner we proposed in Orjuela et al. ${ }^{13}$ in which the depth information of the surface of a carpet is digitize. To scan, the carpet is placed on a drum and on its surface a uniform line is project. The reflection of the line on the surface of the carpet is captured by a camera, which is located at an angle of $45 \%$ degrees and a distance of $30 \mathrm{~cm}$. The angle of $45 \%$ has been chosen to simulate the human visual inspection according to the last EN1471:1996 standard, in 
which the experts evaluate the changes on physical samples placed on a table with this inclination. Additionally, the use of the drum results in a better capturing of the reflection by naturally opening the structure on the surface of the carpet.

A range image is built by rotating the drum, capturing frames spaced $0.24 \mathrm{~mm}$ to cover a surface area with the same pixel size as those in the photographs. We capture five range images for each sample to use equal number of images representing the wear labels as in the set for the intensity images. As a result, each wear labels of the eight reference sets is represented with 10 images: five intensity images and five range images. Samples for both intensity and range images of the carpets used in this approach are shown in Figure 1. In this approach we vary 32 times each image combining complement, rotations of $0,90,180,270$ degrees and mirrored up-down and left-right. These variations have been introduced to add robustness when evaluating the description of the wear labels. Therefore, each sample of a wear label is represented with 320 images (160 intensity images and 160 range images) since there are 5 images per sample in the database.

\section{METHODS}

To evaluate the quality in carpets by rating the appearance retention properties, carpet samples are first treated in a tester to simulate traffic exposure. ${ }^{1}$ Afterwards, the new appearance of the worn samples is rated by humans, who visually compare their changes in pattern, color, hairiness, thickness and structure to samples with original appearance. The change in appearance can be automatically quantified by measuring the change in texture and color in the worn samples compared to the original samples by using image analysis. Particularly, for measuring changes in texture a new method has been recently proposed, which consist in computing the Symmetrized adaptation of the Kullback-Leibler (SKL) divergence ${ }^{19}$ on Local Binary Patterns (LBP) statistics ${ }^{20}$ to estimate the degree of wear of the samples. ${ }^{12}$ In the followings we present a method that improves the LBP technique in discriminating similar textures represented by consecutive wear labels.

We first compute the Rotational, Mirrored and complemented version of the LBP technique (LBPRMC) in the images of the database. The LBPRMC consist in evaluating relationships between points on a circle around a central pixel for each pixel in an image. For this, equally spaced points are considered in the circle. If one of the points on the circle does not lay on the center of a pixel, the corresponding pixel value is interpolated from the four pixels closest to the point. In this approach we use 12 equally spaced circular neighbours in a circle of radius $\sqrt{2}$. Figure 2 illustrates how the circular points are computed.

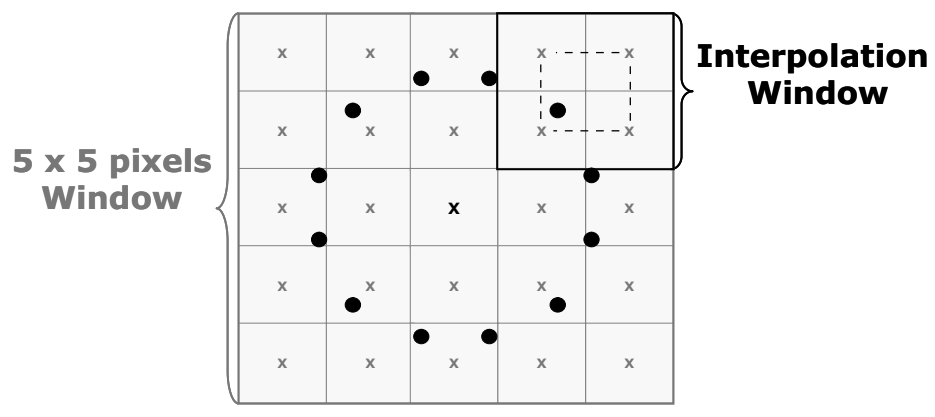

Figure 2: 12 points equally spaced in a circle of $\sqrt{2}$. Points that do not lay on the center of pixels are computed from the closest four pixels by using bilinear interpolation. The graph shows centres of pixels with $\mathbf{x}$. One window of interpolation is highlighted to illustrate the case when a point is non located on a central pixel.

Each one of the points is assigned with a bit by comparing it with the central pixel (1:bigger,0:equal or smaller). The bits are read out clock wise and placed in a diadic code word named pattern. Rotated, mirrored and complemented versions of patterns are grouped using a look up table. Mirror and complement invariance are considered because a carpet reflected in a mirror or with the colors inverted (dark replaced by bright and vice versa) would be assigned the same rating by human assessors. Afterwards, an histogram is built for each image with bins representing the patterns associated with the pixels. Since there were no samples 
of original appearance in the database, the distributions of the LBPRMC patterns corresponding to original appearance were estimated by using a simple linear regression model from the histograms of wear labels 1.0 to 4.5. This was performed considering that patterns are expected to change monotonically from labels 1.0 to 5.0. Within the same assumption, two types of patterns can be identified in the histograms namely 1) patterns that monotonically change with wear labels, 2) patterns randomly changing with wear labels. Grouping patterns type 2 gives a histogram representation more accurate with the change in appearance. We call the resulting patterns the 'Varying Local Binary Patterns' (VLBP). Figure 3 illustrates examples for both types of patterns.

To identify the patterns that are monotonically changing with the wear labels we compute the absolute value of the Spearman rank correlation between the wear labels and the frequency of the bins for each pattern. For this, bins of the same patterns for the 8 wear labels are placed ascendingly by order. The difference between the assigned rank and the expected rank is calculated, assigning a significance between -1 to 1 . 1 indicates a perfect monotonic increasing change and -1 a perfect monotonic decreasing change. We are therefore interested in the absolute value to detect those patterns that are changing monotonically. The Spearman rank correlation is computed as follows:

$$
\rho_{p}=1-\frac{6}{\left(L\left(L^{2}-1\right)\right)} \sum_{l=1}^{L} d_{p l}^{2}
$$

with $L=9$ the number of wear labels and $d_{l}$ the difference between the assigned rank and the expected rank for the frequency of the pattern $p$ in the wear label $l$ (with $l$ from 1 to $L$ ).

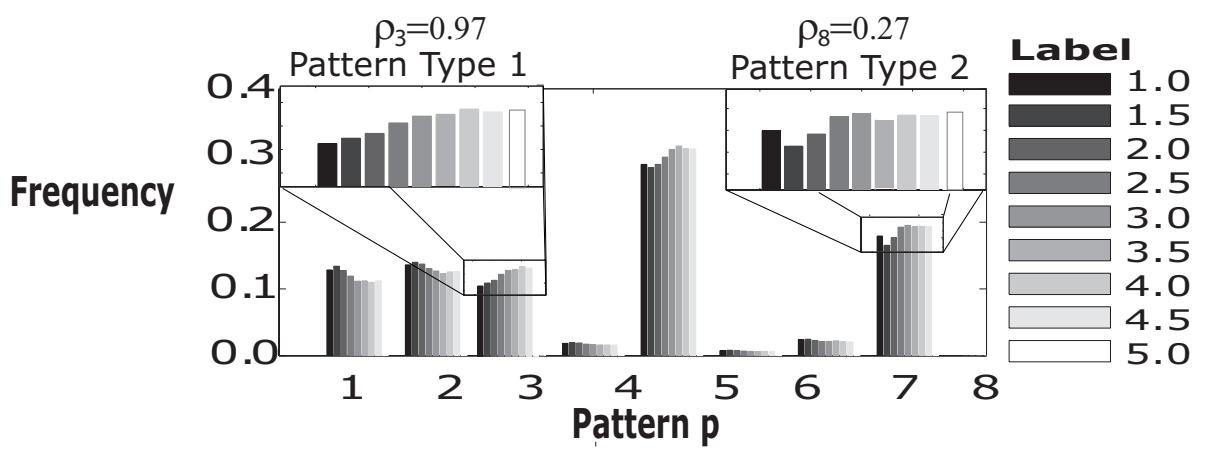

Figure 3: Changing detection of the 8 first bins out of a total of 125 in images of intensity using LBPRMC patterns 12 equally spaced circular neighbours in a circle of radius $\sqrt{2}$.

Since $\rho_{p}=1$ is unlikely to happen, we established a threshold, termed $t$ that permits to differentiate patterns type 1 from patterns type 2 in the whole histogram. This results in maximum texture discriminance between consecutive wear labels by grouping patterns type two in one bin reducing their noise effects on the representation. We quantify the difference between one histogram corresponding to an original appearance and one histogram corresponding to a worn sample for different thresholds until we find the optimal that gives better discrimination between the consecutive wear labels. It is also important that the quantified differences are highly linear rank correlated with the wear labels. To quantify the difference, one SKL value, termed $\kappa$, is computed between one histogram corresponding to an original appearance and one histogram corresponding to a worn sample. The two histograms are normalized into $h_{1}(i)$ and $h_{2}(i), i$ is the bin index and $N$ is the total number of bins. The $\kappa$ value between $h_{1}(i)$ and $h_{2}(i)$ is computed as follows:

$$
\kappa=\sum_{i=1}^{N} h_{1}(i) \log h_{1}(i)+\sum_{i=1}^{N} h_{2}(i) \log h_{2}(i)-\sum_{i=1}^{N}\left(h_{1}(i)+h_{2}(i)\right) \log \left(h_{1}(i)+h_{2}(i)\right)
$$


Some of the $\kappa$ values from the same worn sample can be significantly larger or smaller than the others because of the presence of factors such as flecks or speckles that could not be removed by the vacuum cleaning. These outliers are detected using the Mahalanobis distance and then replaced with the median from the $\kappa$ values of the worn sample to allow statistical comparisons with an equal number of $\kappa$ values per worn sample.

LBPRMC has been show previously to perform better than other extensions of the LBP technique. Therefore, we compare in this approach the performance of the LBPRMC technique against the VLPB technique. Consequently with previous comparisons, we evaluate the performance of the $\kappa$ values describing the degree of wear following the procedure described in Orjuela et al. ${ }^{12}$ For this, two characteristics are quantified from checking the relation between the mean of the $\kappa$ values per wear label and the wear labels. The first characteristic,termed $\omega$, is quantified by measuring the linear-rank correlation, indicating the monotonicity of the relationship. The linear-rank correlation is computed similarly like in 1 but ordering the mean of the $\kappa$ values per wear label. The second characteristic measures the discriminance between $\kappa$ values from consecutive wear labels. For this, we count how many times the difference between consecutive means is larger than the threshold for a statistic significance as follows:

$$
\left(\mu_{(l+1)}-\mu_{l}\right)-\varsigma>0
$$

The significance is computed based on the Tukey test ${ }^{21}$ as follows:

$$
\varsigma=\frac{q_{(\alpha, L, S L-L)}}{\sqrt{(S L-L) S}} \sqrt{\sum_{l=1}^{L} \sum_{s=1}^{S}\left(\kappa_{l s}-\mu_{l}\right)^{2}}
$$

$q_{(\alpha, L, S L-L)}=3.97$ is obtained from the studentized range distribution at $100(1-\alpha)$ of confidence. $S=32$ is the number of $\kappa$ values, $L=9$ is the number of wear labels and $\mu_{l}$ is the mean value of the $\kappa$ values associated with the wear label $l$.

To determine the threshold $(t)$ that differentiate patterns type 1 from patterns type 2 , we look for the highest $\omega$ and $\tau$ (maximum product) when varying $t$. The optimal $t$ value is different for each type of carpet. Figure 4 illustrates one example selecting $t$ for carpet type Frisé. $\tau$ is normalized to facilitate the visualization and scale for the number of bins grouped (NBG) is displayed on the right. The graph shows that the optimal $t$ value for this particular case is found to be in the range of $0.48 \pm 0.015$, increasing the rank correlation between $\kappa$ values and wear labels from 0.9 to 1 as well as increases the efficiency discriminating consecutive wear labels from 0.72 to 1 . The graph shows also that between 70 and 90 patterns can be grouped for a better description. Additionally, the linear correlation $(r)$ performance is shown.

\section{RESULTS}

The performance of the description using SKL values from the LBPRMC technique against SKL values from the VLBP technique are compared using an ANalysis Of Variance test (ANOVA) on $\omega$ and $\tau$ for each carpet type. ANOVA test identifies significant differences between both tests if a computed probability, termed $p_{d}$, is smaller than a given $\alpha$. In that case the techniques are said to be different with a $100(1-\alpha) \%$ of confidence. For valid $p_{d}$-values, the techniques must not interact with the types of images, meaning independence between techniques and type of images. This is inspected by comparing also a probability, termed $p_{i}$ with an $\alpha .^{21}$

The ANOVA results show no interaction between the techniques and the images, $p_{i}(\omega)=0.82$ and $p_{i}(\tau)$ $=0.99$ ). Thus, for both types of images the VLBP technique significantly increases the monotonicity with a confidence of $95 \%\left(p_{d}(\omega)=0.35<0.05\right)$ and the discriminance between consecutive wear labels with a confidence of $99 \%\left(p_{d}(\tau)<0.01\right)$ for both types of images. This is illustrated in Figure 5. 


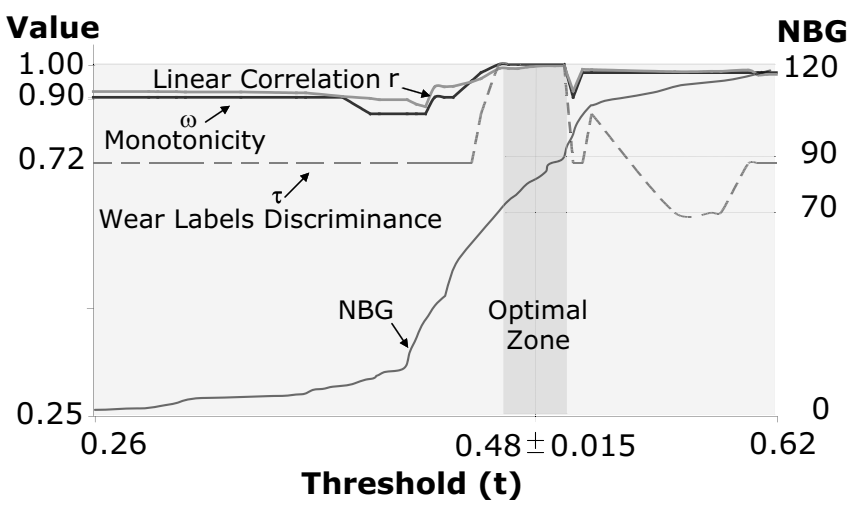

Figure 4: Example of selecting $t$ for carpet type Frisé. The optimal $t$ value for this particular case is found to be in $0.48 \pm 0.015$ for this range, the rank correlation between $\kappa$ values and wear labels is equal to 1 as well as the efficiency discriminating consecutive wear labels is equal to 1 . The graph shows also the performance of the linear correlation $(r)$ between $\kappa$ values and wear labels.

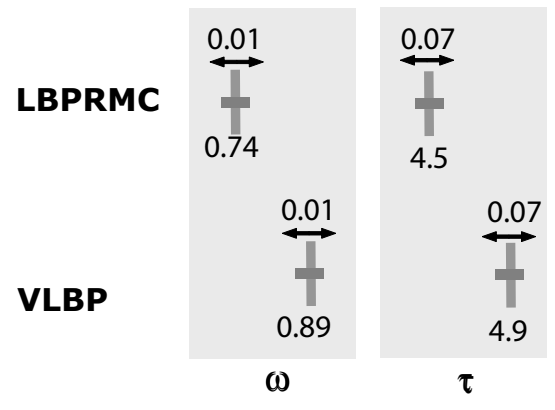

Figure 5: Comparison of the performance of the LBPRMC with the VLP technique in intensity and depth images. The figure shows mean values, at the bottom of the boxes, and standard deviations, represented with $\leftrightarrow$, at the top of the boxes.

\section{CONCLUSIONS}

In this paper a new extension of the LBP technique, termed Varying Local Binary Patterns (VLBP) for better discriminating consecutive wear labels has been presented. The VLBP consist in detecting those LBPRMC (Rotated, Mirrored and Complemented versions) patterns that monotonically change with the wear labels. The other patterns are grouped. To differentiate both types of patters an automatic threshold is computed based on evaluating the performance of the VLBP patterns for different thresholds. The performance is evaluated on the description given, comparing the pattern histogram obtained from original samples to worn samples by computing the simetric Kullback-Leibler divergence. The new technique has been tested in both intensity and range images of samples from the EN1471:1996 standard, covering 72\% of the existing references in Europe. Results show that grouping the non monotonic patterns significantly increases the monotonicity in the representation as well as the discrimination between consecutive wear labels.

\section{REFERENCES}

[1] American Society for Testing and Materials. 2010 Annual Book of ASTM Standards, General Methods and Instrumentation, volume 14.01-14.04. ASTM International, 2010.

[2] The Carpet and Rug Institute. Assessment of carpet surface appearance change using the cri reference scales. Technical Bulletin, 2003.

[3] European Committee for standardization. Constructional details of types of textile floor covering available as reference fatigued specimens, 1996. 
[4] L. H. Siew, R. M. Hodgson, and E. J. Wood. Texture measures for carpet wear assessment. IEEE Transactions on Pattern Analysis and Machine Intelligence, 10:92 - 105, 1988.

[5] E. Wood and R. Hofgson. Carpet texture measurement using image analysis. Textile Research Journal, 59:1-12, 1989.

[6] Y. Wu, B. Pourdeyhimi, and S. M. Spivak. Texture evaluation of carpets using image analysis. Textile Research Journal, 61:407-419, July 1991.

[7] J. Sobus, B. Pourdeyhimi, J. Gerde, and Ulcay Y. Assessing changes in texture periodicity due to appearance loss in carpets: Gray level co-occurrence analysis. Textile Research Journal, 61:557-567, October 1991.

[8] B. Xu. Assessing carpet appearance retention by image analysis. Textile Research Journal, 64:497-509, 1994.

[9] J. Wang and E. J. Wood. A new method for measuring carpet texture change. Textile Research Journal, 65:196-202, April 1994.

[10] S. Sette, L. Boullart, and P. Kiekens. Self-organizing neural nets: A new approach to quality in textiles. Textile Research Journal, 65:196-202, April 1995.

[11] W. Van Steenlandt, D. Collet, S. Sette, P. Bernarn, R. Luning, L. Teze, H. Bohland, and H. Schulz. Automatic assesment of carpet wear using image analysis and neural networks. Textile Research Journal, 66(9):555-561, 1996.

[12] S. A. Orjuela, E. Vansteenkiste, F. Rooms, S. De Meulemeester, R. De Keyser, and W. Philips. Evaluation of the wear label description in carpets by using local binary pattern techniques. Textile Research Journal, Acepted July, 2010.

[13] S. A. Orjuela, E. Vansteenkiste, F. Rooms, S. De Meulemeester, R. De Keyser, and W. Philips. Feature extraction of the wear label of carpets by using a novel 3d scanner. In Proc. of the Optics, Photonics and Digital Technologies for Multimedia Applications conference, 2010.

[14] S. A. Orjuela, E. Vansteenkiste, F. Rooms, S. De Meulemeester, R. De Keyser, and W. Philips. A comparison between intensity and depth images for extracting features related to wear labels in carpets. In Proc. of the SPIE 7798, 2010.

[15] S. A. Orjuela, E. Vansteenkiste, F. Rooms, S. De Meulemeester, R. De Keyser, and W. Philips. Texture wear analysis in textile floor coverings by using depth information. In Proc. of the XIII Simposio de Tratamiento de señales, Imágenes y Visión Artificial, 2010.

[16] S. A. Orjuela, B. Ortiz, S. J. De Meulemeester, C. Garcia, F. Rooms, A. Pizurica, and W. Philips. Surface reconstruction of wear in carpets by using a wavelet edge detector. In In Proc. of Advanced Concepts for Intelligence Vision Systems. Accepted August, 2010.

[17] T. Ojala, Pietikainen M., and Menp T. Multiresolution gray scale and rotation invariant texture classification with local binary patterns. IEEE Transactions on Pattern Analysis and Machine Intelligence, 24(7):971-987, July 2002.

[18] R. C. Gonzalez and R. E. Woods. Digital Image Processing. Prentice Hall, 2 edition, January 2002.

[19] M. Petrou and P. G. Sevilla. Image Processing Dealing with Texture. Wiley, January 2006.

[20] M. Ojala, T. Pietikainen and Z. Xu. Rotation-invariant texture classification using feature distributions. Pattern Recognition, 33(1):43-52, January 2000.

[21] M. Kutner, C. J. Nachtsheim, J. Neter, and Li W. Applied Linear Statistical Models. McGraw-Hill/Irwin, 5 edition, 2004. 